\title{
Modélisation de l'interception des rayonnements solaires dans une culture en rangs. II. Structure géométrique du couvert et validation du modèle
}

\author{
H. Sinoquet et R. Bonhomme \\ INRA Antilles-Guyane, Station agropédoclimatique, Unité de bioclimatologie, BP 1232, F97184 Pointe-à-Pitre Cedex, \\ France (Antilles)
}

(reçu le 30 octobre 1987, accepté le 13 mars 1989)

\begin{abstract}
Résumé - Dans une première partie, un modèle de transferts radiatifs appliqué à une culture en rangs a été décrit et testé sur des couverts homogènes. Pour effectuer la validation dans le cas de cultures en rang, des mesures de rayonnement réfléchi et transmis au sol ont été réalisées sur 2 parcelles de maīs dont les rangs étaient orientés est-ouest (E-O) pour l'une et nord-sud $(\mathrm{N}-\mathrm{S})$ pour l'autre. La structure géométrique, mesurée grâce à la méthode des silhouettes, est différente selon l'orientation des rangs. La répartition des azimuts des plantes n'est pas uniforme. Celle des inclinaisons des feuilles est globalement uniforme, mais présente des variations spatiales. Quant à la densité de surface foliaire, elle montre de grandes variations dans le plan horizontal, en fonction de la distance au centre du rang, même avec une culture bien développée. L'ajustement des flux radiatifs calculés par le modèle aux mesures, évalué par régression linéaire, est satisfaisant et ne présente pratiquement pas de variations en fonction de l'orientation des rangs. Les erreurs quadratiques moyennes varient de 10 à $20 \%$ en fonction du type de rayonnement. Les valeurs optimisées de l'indice de dispersion du feuillage (Nilson, 1971) indiquent un comportement agrégatif qui tend à diminuer au cours du développement de la culture, notamment pour l'orientation N-S. L'agencement est beaucoup moins agrégatif dans les rangs orientés $\mathrm{E}-\mathrm{O}$.
\end{abstract}

distribution spatiale du feuillage - orientation des rangs - Zea mays L. - paramètre d'agencement du feuillage

Summary - Modelling of solar radiation interception in row crops. II. Crop geometry and validation of the model. A radiative transfer model applied to a row crop has previously been described and tested on homogeneous canopies. To validate this model for row crops, measurements of reflected and transmitted radiation were made on two maize canopies : one orientated East-West, and the other North-South. The geometrical structure, measured with the plant profile method, differs according to row orientation. The plant azimuth distribution is not uniform. That of leaf inclination is globally uniform, but it presents spatial variations. The leaf area density shows large variations in the horizontal plane, depending on the distance from the center of the row, even in the case of a well developed crop. Linear regressions show a good agreement between calculated and measured values, and are quite similar for both row orientations. The mean quadratic errors are from $10-20 \%$, depending on the nature of the radiation. Optimized values of leaf dispersion index (Nilson, 1971) indicate a clumped behaviour which decreases with the development of the canopy (mainly for the North-South orientation), with however a more clumped arrangement in the North-South rows.

spatial distribution of leaf area - row direction - Zea mays L. - leaf dispersion index

\section{Introduction}

La détermination de la photosynthèse et de la transpiration d'une culture nécessite le calcul de la répartition des rayonnements dans la végétation. Dans ce but, des modèles mathématiques décrivant les interactions entre rayonnement et végétation ont été développés. Ces modèles peuvent être classés en 2 groupes (Lemeur et
Blad, 1974) : d'une part, les modèles géométriques qui s'appliquent à un ensemble de formes distribuées régulièrement dans l'espace, et qui sont surtout utilisables pour de grandes échelles d'espace et de temps; d'autre part, les modèles statistiques dans lesquels le couvert végétal est représenté par des distributions décrivant l'arrangement des éléments de feuillage de la culture. C'est un modèle de ce type qui a été présenté 
dans la première partie de cette étude (Sinoquet, 1989) pour décrire les échanges radiatifs dans une culture en rangs, c'est-à-dire une culture hétérogène selon une direction du plan horizontal. Dans cette approche, la structure géométrique du couvert est traduite en termes de facteurs de forme, et le bilan radiatif de la culture est résolu par une méthode dérivée de la méthode des radiosités (Ozisik, 1981).

Dans la première partie, le modèle a été testé sur des mesures effectuées sur un couvert supposé homogène, et il a été comparé à un modèle de transferts radiatifs applicable dans ces conditions (Bonhomme et Varlet-Grancher, 1977). Ces résultats, qui ont montré la cohérence de la méthode proposée, ne permettent pas d'affirmer un bon fonctionnement du modèle lors de son application à une culture hétérogène. Dans cette seconde partie de l'étude, nous présentons une première validation du modèle sur un couvert spatialement hétérogène. Elle est réalisée à partir de mesures des rayonnements réfléchi et transmis par 2 cultures de maïs en rangs, dont la structure géométrique a été mesurée.

\section{Matériel et Méthodes}

\section{Dispositif cultural}

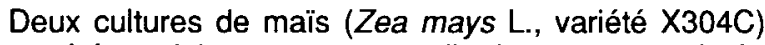
ont été conduites sur une parcelle de $25 \times 50 \mathrm{~m}$, située à la Guadeloupe sur le domaine du Centre des Antilles de l'Institut National de la Recherche Agronomique $\left(16^{\circ} 15^{\prime} \mathrm{N}, 61^{\circ} 40^{\prime} \mathrm{W}\right)$. Dans le premier essai (févriermars 1986), les plantes ont été semées selon des rangs orientés est-ouest $(E-O)$, alors que les rangs étaient orientés selon la direction nord-sud $(\mathrm{N}-\mathrm{S})$ dans le second essai (mai-juin 1986). Pour les 2 cultures, l'écartement entre 2 rangs était de $0,8 \mathrm{~m}$ et la distance entre 2 plantes sur le rang était de $0,25 \mathrm{~m}$, ce qui correspond à une densité de 5 plantes. $\mathrm{m}^{-2}$. Les conditions de culture (fertilisation, suivi phytosanitaire...) correspondaient aux pratiques agronomiques appliquées classiquement à ce type de culture en milieu tropical.

\section{Mesures de rayonnement}

Des mesures de rayonnement incident, réfléchi par la culture et transmis au sol, ont été effectuées de la levée à la floraison dans le domaine de longueurs d'onde du rayonnement solaire (250 à $2500 \mathrm{~nm}$ ) avec des pyranomètres (Kipp et Zonen) et dans le domaine visible (400 à $700 \mathrm{~nm}$ ) avec des Quantum Sensors (LiCor Inc.). Seul le rayonnement global $\mathrm{G}$ était mesuré au-dessus du couvert : la répartition entre rayonnements direct $S$ et diffus $D$ a donc été estimée par une relation linéaire établie dans nos conditions à l'échelle horaire (Sinoquet, 1985) :

$$
(D / G)=1,12-1,20\left(G / G_{0}\right) \quad r^{2}=0,76 \quad N=2308
$$

où $G_{0}$ est le rayonnement extraterrestre. Bien que la corrélation entre ces 2 variables soit assez élevée, l'erreur d'estimation du rapport (D/G) reste importante puisqu'elle atteint $11 \%$.
Pour les rayonnements réfléchis, les capteurs étaient fixés sur un mât à $3 \mathrm{~m}$ de hauteur, surface réceptrice orientée vers le bas. Les capteurs de rayonnement transmis étaient fixés sur un chariot mobile de $2 \mathrm{~m}$ de longueur, situé sous la culture et perpendiculairement à la direction des rangs. Ce dispositif permettait d'intégrer spatialement le rayonnement transmis par la culture. Les capteurs étaient reliés à un centralisateur de mesures CR21 Micrologger (Campbell Scientific Inc.) effectuant une scrutation par minute et des moyennes horaires.

\section{Mesure de la structure géométrique du couvert}

La description de la structure géométrique du couvert a été réalisée grâce à la méthode des silhouettes (Bonhomme et Varlet-Grancher, 1978). Utilisée initialement pour décrire des couverts homogènes dans le plan horizontal, elle a été étendue à une description en 2 dimensions de la culture. Pour une journée donnée, 12 plantes sont prélevées au champ, placées contre une surface plane graduée et photographiées. Les clichés sont numérisés en assimilant chaque feuille à une suite de segments dont la longueur et l'inclinaison sont déduites des coordonnées $(x, y)$ de leurs extrémités. La détermination de la surface du segment nécessite la connaissance de l'évolution de la largeur des feuilles en fonction de la distance au point d'insertion sur la tige, qui est décrite par une relation polynômiale, déterminée pour chaque variété. Pour la variété utilisée $(X 304 C)$, l'ajustement suivant est satisfaisant :

$$
\begin{aligned}
& 1 / I_{\max }=0,882-1,332\left(L / L_{t o t}\right)+8,274\left(L L_{t o t}\right)^{2} \\
& -12,763\left(L L_{\text {tot }}\right)^{3}+4,925\left(L L_{\text {tot }}\right)^{4} \quad r^{2}=0,98 \quad N=243
\end{aligned}
$$

où I est la largeur de la feuille à une distance $L$ du point d'insertion sur la tige, $I_{\max }$ est la largeur maximale de la feuille et $L_{\text {tot }}$ est la longueur totale de la feuille. L'utilisation de cette relation nécessite donc la seule mesure de la largeur maximale de chaque feuille des plantes photographiées. La longueur totale est, quant à elle, déterminée par la somme des longueurs de l'ensemble des segments. Compte tenu de l'erreur d'estimation du rapport $\left(1 / /_{\max }\right)$, la surface foliaire de chaque feuille est calculée avec une précision de l'ordre de $6 \%$.

Chaque segment de feuille est alors classé dans la couche horizontale, la tranche verticale et la classe d'inclinaison auxquelles il appartient, et cela en tenant compte de l'azimut de la plante, mesuré au champ (entre $0^{\circ}$ et $90^{\circ}$ ) par rapport à la direction du rang : les feuilles perpendiculaires aux rangs (azimut $=90^{\circ}$ ) s'étaleront dans un nombre de tranches verticales maximum, alors que les feuilles situées dans le plan du rang (azimut $=0^{\circ}$ ) n'occuperont que la tranche centrée sur le rang. Cette méthode permet d'obtenir, pour chaque tube $k$, la densité de surface foliaire $L_{k}\left(m^{2}\right.$ de surface foliaire par $\mathrm{m}^{3}$ ) et la distribution des inclinaisons $g_{k}(\alpha)$. Elle n'est cependant utilisable que pour des plantes ayant leurs feuilles dans un même plan vertical; c'est théoriquement le cas du maïs, bien que cette condition ne soit pas toujours parfaitement respectée au champ, notamment à cause de l'action du vent.

La structure géométrique du couvert a été mesurée à 5 reprises pour la culture orientée $\mathrm{E}-\mathrm{O}$ et 4 fois pour la culture $N-S$. Ces mesures vont du stade « 9 feuilles visibles" à la floraison, ce qui correspond à des indices foliaires de 0,3 à 2,7 (Tableau I). 
Tableau I. Caractéristiques des cultures aux jours où la structure géométrique a été mesurée.

\begin{tabular}{|c|c|c|c|c|}
\hline Date & $J A L$ & IF & NFV & $N F D$ \\
\hline \multicolumn{5}{|c|}{ Culture orientée est-ouest } \\
\hline 24.2 .86 & 31 & $0,33( \pm 0,05)$ & $9,0( \pm 0,2)$ & $6,8( \pm 0,2)$ \\
\hline 4.3 .86 & 39 & $0,73( \pm 0,11)$ & $11,2( \pm 0,3)$ & $8,4( \pm 0,2)$ \\
\hline 10.3.86 & 45 & $1,14( \pm 0,16)$ & $12,7( \pm 0,4)$ & $10,4( \pm 0,3)$ \\
\hline 17.3 .86 & 52 & $1,77( \pm 0,19)$ & $15,0( \pm 0,4)$ & $12,8( \pm 0,4)$ \\
\hline 24.3.86 & 59 & $2,17( \pm 0,20)$ & $16,4( \pm 0,5)$ & $15,8( \pm 0,5)$ \\
\hline \multicolumn{5}{|c|}{ Culture orientée nord-sud } \\
\hline 20.5 .86 & 30 & $0,89( \pm 0,09)$ & $11,8( \pm 0,2)$ & $8,9( \pm 0,1)$ \\
\hline 26.5 .86 & 36 & $1,55( \pm 0,13)$ & $14,4( \pm 0,3)$ & $10,6( \pm 0,2)$ \\
\hline 2.6 .86 & 43 & $2,27( \pm 0,15)$ & $16,9( \pm 0,2)$ & $13,4( \pm 0,3)$ \\
\hline 9.6 .86 & 50 & $2,72( \pm 0,17)$ & $18,6( \pm 0,3)$ & $17,0( \pm 0,6)$ \\
\hline
\end{tabular}

JAL : nombre de jours après la levée; IF : indice foliaire; NFV : nombre de feuilles visibles; NFD : nombre de feuilles dégainées. Les valeurs entre parenthèses correspondent aux intervalles de confiance au seuil de $5 \%$.

\section{Structure géométrique du couvert}

\section{Distribution des azimuts des plantes}

Comme les feuilles d'un plant de maïs sont supposées être contenues dans un même plan vertical, la distribution des azimuts des plantes est, en première approximation, identique à celle des azimuts des feuilles. La distribution des azimuts des plantes par rapport à la direction des rangs a été mesurée en considérant une gamme de variation entre 0 et $90^{\circ}$, c'est-à-dire en supposant une répartition égale de la surface foliaire de part et d'autre de la tige (Tableau II). Le test du $\chi^{2}$ indique que seule la distribution azimutale de la culture orientée E-O est significativement différente de la distribution uniforme adoptée dans le modèle.

Les écarts à la distribution uniforme sont dus en partie à une faible représentation de la classe centrée sur $0^{\circ}$, tendance que l'on peut également observer sur la culture orientée N-S. Une simple question d'encombrement de l'espace permet d'expliquer cette situation. Des observations

Tableau II. a. Fréquences des azimuts des plantes, par rapport à la direction des rangs. b. Comparaison des distributions réelles à la distribution uniforme : contribution de chaque classe au $\chi^{2}$. c. Comparaison des 2 distributions réelles : contribution de chaque classe au $\chi^{2}$.

\begin{tabular}{lcccc} 
Classes & & & \\
d'azimut (degrés) & $0-15$ & $15-45$ & $45-75$ & $75-90$ \\
$\begin{array}{l}\text { Culture est-ouest } \\
\text { (60 plantes) }\end{array}$ & 0,07 & 0,27 & 0,38 & 0,28 \\
$\begin{array}{l}\text { Culture nord-sud } \\
\begin{array}{l}(48 \text { plantes) } \\
\text { Distribution uniforme }\end{array}\end{array}$ & 0,08 & 0,33 & 0,42 & 0,17 \\
\hline
\end{tabular}

\begin{tabular}{|c|c|c|c|c|c|}
\hline Classes & & & & & Total \\
\hline d'azimut (degrés) & $0-15$ & $15-45$ & $45-75$ & $75-90$ & \\
\hline Culture est-ouest & 3,60 & 0,80 & 0,45 & 4,90 & 9,75 \\
\hline Culture nord-sud & 2,00 & 0,00 & 1,00 & 0,00 & 3,00 \\
\hline \multicolumn{5}{|c|}{ Valeur $d u \chi^{2}(d d l=3, \alpha=5 \%)$} & 7,81 \\
\hline
\end{tabular}

\begin{tabular}{|c|c|c|c|c|c|}
\hline Classes & & & & & Total \\
\hline d'azimut (degrés) & $0-15$ & $15-45$ & $45-75$ & $75-90$ & \\
\hline Culture est-ouest & 0,04 & 0,18 & 0,03 & 9,02 & 9,27 \\
\hline Culture nord-sud & 0,06 & 0,22 & 0,04 & 0,27 & 0,59 \\
\hline Total & 0,10 & 0,40 & 0,07 & 9,29 & 9,86 \\
\hline
\end{tabular}

Valeur du $\chi^{2}(d d l=3, \alpha=5 \%)$ 
similaires ont été faites sur maïs et sorgho (Ross et Nilson, 1967a; Loomis et Williams, 1969); elles ont été attribuées à une adaptation des plantes à la distribution du rayonnement dans la culture, à la direction des rangs ou à la densité de plantation.

D'autre part, le test du $\chi^{2}$ indique que les distributions azimutales des 2 cultures sont significativement différentes (Tableau Ilc). L'écart est essentiellement dû à la classe $90^{\circ}$, qui est beaucoup plus représentée dans la culture E-O. La recherche de la lumière, liée à la course du soleil par rapport à la direction des rangs, pourrait expliquer ce comportement. D'autre part, la direction des vents dominants (alizés d'est), parallèle aux rangs de la culture $\mathrm{E}-\mathrm{O}$, pourrait provoquer un étalement des feuilles dans les interrangs (Mure, 1984).

\section{Distribution des inclinaisons des feuilles}

La Figure 1 montre l'évolution de la distribution des inclinaisons des feuilles au cours du développement de la végétation. Pour les 2 orientations, les distributions sont pratiquement uniformes, avec une légère dominance des inclinaisons élevées. L'interception du rayonnement par la culture dépend aussi de la répartition dans l'espace de la distribution des inclinaisons;


Fig. 1. Répartition de la surface foliaire (avec intervalles de confiance) en fonction de l'inclinaison des feuilles. a. Culture est-ouest. b. Culture nord-sud. par exemple, des feuilles érigées dans les couches supérieures, et horizontales dans les couches inférieures, favorisent une répartition homogène dans l'espace du rayonnement absorbé par le couvert (Bonhomme, 1983).

La répartition spatiale de l'inclinaison des feuilles a été étudiée en divisant un rang de végétation en 4 secteurs de même taille : 2 couches horizontales et 2 tranches verticales, l'une centrée sur le rang, l'autre sur l'interrang (Tableau III). Les fortes inclinaisons $\left(60\right.$ à $\left.90^{\circ}\right)$ sont surtout présentes dans la couche supérieure et au centre des rangs; cette position correspond en effet à l'emplacement du cornet, où les feuilles sont souvent très érigées. Dans l'interrang, les faibles inclinaisons sont fortement représentées, aux dépens des inclinaisons élevées. Cependant, dans chaque tranche verticale, on tend vers la situation idéale décrite plus haut : les inclinaisons sont plus faibles dans la couche inférieure que dans la couche supérieure. Par ailleurs, il n'y a aucune différence significative entre les répartitions spatiales des inclinaisons des 2 cultures.

\section{Distribution spatiale de la surface foliaire}

La répartition dans l'espace de la surface foliaire n'est pas uniforme, que ce soit selon les directions verticale (Fig. 2) ou horizontale (Fig. 3).
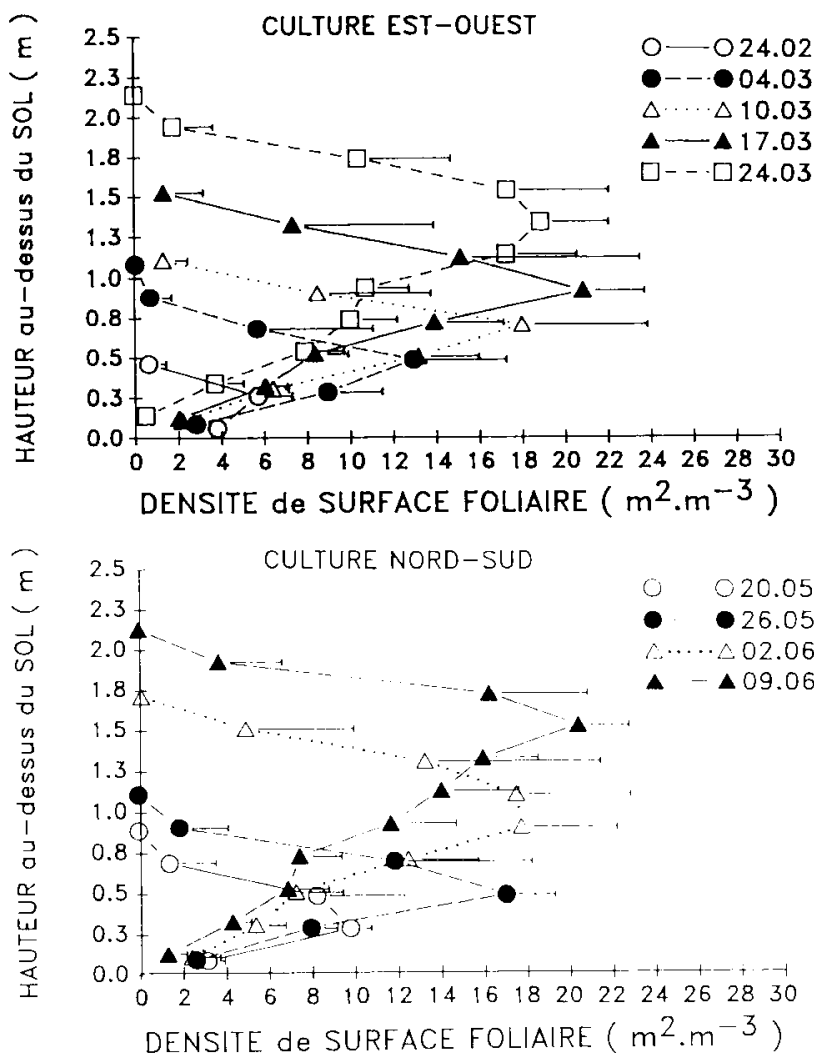

Fig. 2. Profil vertical de densité de surface foliaire (avec intervalles de confiance). a. Culture est-ouest. b. Culture nordsud. 
Tableau III. Répartition spatiale de l'inclinaison des feuilles.

\begin{tabular}{|c|c|c|c|c|c|c|c|}
\hline Classes & $0-30^{\circ}$ & $30-60^{\circ}$ & $60-90^{\circ}$ & $0-30^{\circ}$ & $30-60^{\circ}$ & $60-90^{\circ}$ & \\
\hline \multirow[t]{2}{*}{ Couche supérieure } & $\begin{array}{r}0,03 \\
( \pm 0,02)\end{array}$ & $\begin{array}{r}0,33 \\
( \pm 0,11)\end{array}$ & $\begin{array}{c}0,64 \\
( \pm 0,15)\end{array}$ & $\begin{array}{r}0,36 \\
( \pm 0,15)\end{array}$ & $\begin{array}{r}0,45 \\
\pm 0,16)\end{array}$ & $\begin{array}{c}0,19 \\
( \pm 0,08)\end{array}$ & (1) \\
\hline & $\begin{array}{r}0,04 \\
( \pm 0,03)\end{array}$ & $\begin{array}{r}0,35 \\
( \pm 0,09)\end{array}$ & $\begin{array}{c}0,61 \\
( \pm 0,20)\end{array}$ & $\begin{array}{r}0,34 \\
( \pm 0,14)\end{array}$ & $\begin{array}{c}0,53 \\
( \pm 0,13)\end{array}$ & $\begin{array}{c}0,13 \\
\pm 0,04)\end{array}$ & (2) \\
\hline \multirow[t]{2}{*}{ Couche inférieure } & $\begin{array}{c}0,11 \\
( \pm 0,06)\end{array}$ & $\begin{array}{c}0,60 \\
( \pm 0,09)\end{array}$ & $\begin{array}{r}0,29 \\
( \pm 0,14)\end{array}$ & $\begin{array}{r}0,52 \\
( \pm 0,15)\end{array}$ & $\begin{array}{c}0,31 \\
( \pm 0,13)\end{array}$ & $\begin{array}{r}0,17 \\
( \pm 0,06)\end{array}$ & (1) \\
\hline & $\begin{array}{r}0,14 \\
( \pm 0,05)\end{array}$ & $\begin{array}{c}0,57 \\
( \pm 0,08)\end{array}$ & $\begin{array}{c}0,29 \\
( \pm 0,12)\end{array}$ & $\begin{array}{c}0,55 \\
( \pm 0,18)\end{array}$ & $\begin{array}{c}0,34 \\
( \pm 0,12)\end{array}$ & $\begin{array}{c}0,11 \\
( \pm 0,03)\end{array}$ & (2) \\
\hline Classes & $0-30^{\circ}$ & $30-60^{\circ}$ & $60-90^{\circ}$ & & & & \\
\hline \multirow[t]{2}{*}{ Ensemble de la culture } & $\begin{array}{r}0,21 \\
( \pm 0,05)\end{array}$ & $\begin{array}{c}0,41 \\
( \pm 0,08)\end{array}$ & $\begin{array}{r}0,38 \\
( \pm 0,07)\end{array}$ & (1) & & & \\
\hline & $\begin{array}{c}0,21 \\
( \pm 0,04)\end{array}$ & $\begin{array}{r}0,44 \\
( \pm 0,05)\end{array}$ & $\begin{array}{r}0,35 \\
( \pm 0,09)\end{array}$ & (2) & & & \\
\hline
\end{tabular}

La végétation, sur un interrang, est divisée en 4 secteurs de même taille : horizontalement, une couche inférieure et une couche supérieure; verticalement, une tranche de $40 \mathrm{~cm}$ centrée sur le rang, l'autre centrée sur l'interrang. Pour chaque secteur sont indiqués les pourcentages de surface foliaire contenue dans les classes d'inclinaisons $0-30^{\circ}, 30-60^{\circ}, 60-90^{\circ}$, pour les cultures est-ouest (1) et nord-sud (2) en fin de période végétative. Les valeurs entre parenthèses correspondent aux intervalles de confiance au seuil de $5 \%$.
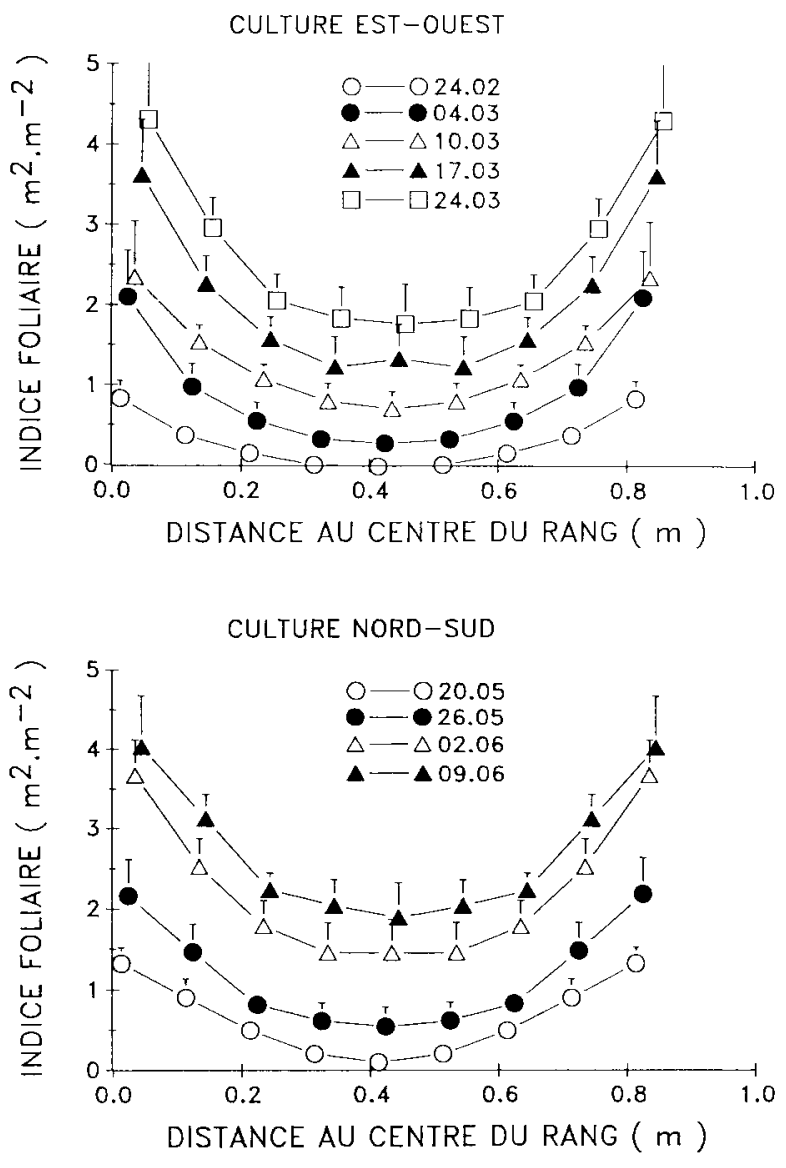

Fig. 3. Profil horizontal d'indice foliaire. a. Culture est-ouest. b. Culture nord-sud.
La forme des profils verticaux de densité de surface foliaire est classique (Ross et Nilson, 1967b) et les variations qui peuvent être observées dépendent notamment de l'inclinaison des feuilles et de leur niveau d'insertion sur la tige (Myneni et al., 1986). La diminution de la surface foliaire dans les couches inférieures au cours du développement de la végétation est due au dessèchement des premières feuilles (Ross et Nilson, 1967b).

Bien que cela soit souvent négligé dans les modèles de tranisferts radiatifs, la densité de surface foliaire varie fortement le long d'un axe horizontal perpendiculaire à la direction des rangs; Ross (1981) et Myneni et al. (1986) rapportent des faits identiques. Dans les premiers stades de végétation, une partie de l'interrang est pratiquement vide; en fin de période végétative, la culture, qui semble homogène à première vue, est en fait 2 à 2,5 fois plus dense au centre du rang qu'au centre de l'interrang. $A$ indice foliaire équivalent, la variation horizontale de densité foliaire est moins accentuée dans le cas des rangs orientés $\mathrm{E}-\mathrm{O}$, ce qui découle directement des observations faites sur la distribution des azimuts des plantes. Cette tendance s'atténue cependant pour les indices foliaires (IF) croissants : pour la culture orientée $\mathrm{N}-\mathrm{S}$, le rapport entre densité de surface foliaire au centre du rang et au centre de 
l'interrang varie de 13 pour $\mathrm{IF}=0,9$ à 4,1 pour $\mathrm{IF}=1,6$ et 2,56 pour $\mathrm{IF}=2,3$; pour des indices foliaires similaires, les valeurs du rapport passent de 7,3 à 2,7 et 2,45 pour les rangs orientés E-O. La distribution de la surface foliaire selon l'axe horizontal parallèle aux rangs n'a pas été étudiée; dans la modélisation, elle est supposée uniforme, bien que Myneni et al. (1986) observent une certaine hétérogénéité selon cette direction.

\section{Validation du modèle}

La validation a consisté à comparer les rayonnements, réfléchis et transmis au sol, mesurés dans les cultures à ceux calculés par le modèle. Rappelons que ce modèle a été décrit dans la première partie de cette étude (Sinoquet, 1989). Les mesures utilisées sont les moyennes horaires fournies par la centrale d'acquisition entre $7 \mathrm{~h}$ et 17h. Elles correspondent aux dates où la structure du couvert a été déterminée ainsi qu'à la veille et au lendemain de ces journées, en supposant que la structure géométrique n'avait pas évolué. Seules les mesures indiquant nettement un mauvais fonctionnement des capteurs (valeurs négatives ou supérieures au rayonnement incident) ont été écartées.

\section{Valeurs des paramètres d'entrée}

Les simulations ont été effectuées en supposant que la moitié du rayonnement solaire incident appartient au domaine visible (400 à $700 \mathrm{~nm}$ ), l'autre moitié au domaine proche infrarouge (700 à $1200 \mathrm{~nm}$ ) (Varlet-Grancher, 1975). Les propriétés optiques des feuilles ont été supposées constantes dans chacune de ces 2 gammes de longueurs d'onde; les valeurs du facteur de réflexion des feuilles $r_{F}$ qui ont été choisies sont : $r_{F}$ (vis) $=0,05$ et $r_{F}$ (pir) $=0,40$.

Dans le cas des sols ferrallitiques de Guadeloupe, l'albédo varie entre 0,05 et 0,09 , en fonction de l'humidité de surface du sol. Celle-ci n'ayant pas été contrôlée, une valeur moyenne de 0,07 a été retenue, et cela pour les 2 domaines de longueur d'onde.

Les valeurs utilisées pour les paramètres de discrétisation sont les suivantes : $10^{\circ}$ pour les classes d'inclinaison et de hauteur, $45^{\circ}$ pour les classes d'azimut, $1 \mathrm{~cm}$ pour le pas d'espace du maillage dans le cas de l'interception des rayonnements direct et diffus. La végétation était représentée par 3 couches horizontales de même épaisseur, ajustée à la taille des plantes, et par 4 tranches verticales de $0,2 \mathrm{~m}$ pour la distance entre 2 rangs. Une telle discrétisation de l'espace occupé par la végétation permet de tenir compte des variations spatiales de la structure géométrique du couvert, sans alourdir les calculs géométrique du couvert, sans alourdir les calculs de façon exagérée : la culture est décrite par 12 tubes de végétation. De plus, une description plus fine du couvert ne serait pas très réaliste : dans ce cas, la précision sur les paramètres de structure diminuerait rapidement, à cause de leur multiplication.

Seul le paramètre d'agencement des feuilles (Nilson, 1971) a servi de paramètre de calage du modèle. II est supposé être identique pour tous les tubes de végétation, et des valeurs comprises entre 0 et $-3,5$ ont été testées. Pour chaque date, la valeur permettant d'obtenir le meilleur ajustement des flux calculés aux flux mesurés a été retenue. Cette gamme de variation correspond à des agencements agrégatifs; cela semble être le cas du maïs, car les feuilles sont en première approximation dans un même plan vertical et ont donc tendance à être superposées.

\section{Comparaison des rayonnements calculés et mesurés}

\section{Comparaison des rayonnements réfléchis}

Les rayonnements réfléchis calculés et mesurés sont présentés à la Figure $4 a$ et b pour les rayonnements global et visible. Toutes mesures confondues, l'ajustement des calculs aux mesures est satisfaisant dans le cas du rayonne-

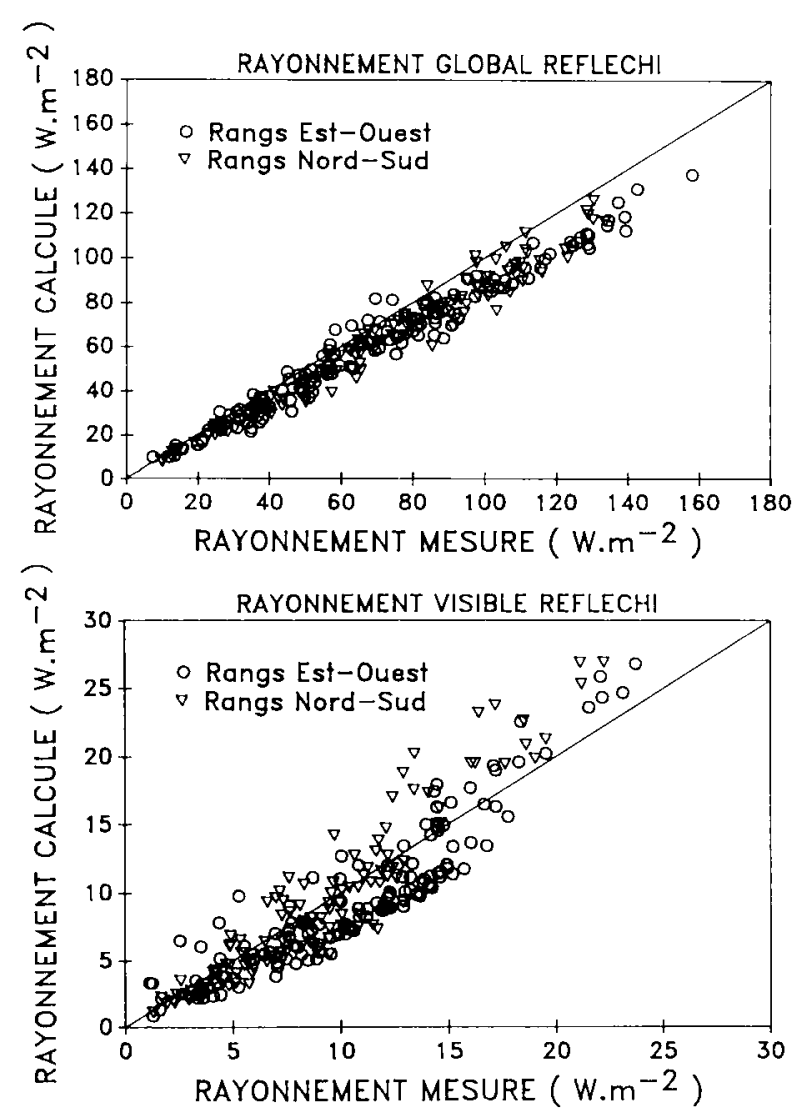

Fig. 4. Comparaison des rayonnements réfléchis calculés et mesurés. a. Rayonnement global. b. Rayonnement visible. 
ment global. La droite de régression entre valeurs mesurées et calculées (Tableau IV) est proche de la première bissectrice, bien que sa pente soit significativement différente de 1 : elle indique une légère sous-estimation. Cependant, l'erreur moyenne (définie comme la racine carrée de la moyenne des carrés des écarts mesuremodèle) est égale à $11 \mathrm{~W} \cdot \mathrm{m}^{-2}$, ce qui correspond à un niveau de rayonnement très faible.

Dans le cas du rayonnement visible réfléchi, la dispersion des points autour de la première bissectrice est plus importante (Fig. 4b), ce que confirme la valeur plus faible du coefficient de détermination de la droite de régression (Tableau IV). Cependant, l'erreur moyenne est inférieure à $3 \mathrm{~W} \cdot \mathrm{m}^{-2}$. Cette valeur est à la limite de l'erreur de mesure, compte tenu de la précision du capteur et de la résolution du centralisateur de mesures. D'autre part, la pente de la droite de régression (respectivement l'ordonnée à l'origine) n'est pas significativement différente de 1 (respectivement de 0).

\section{Comparaison des rayonnements transmis}

Les rayonnements transmis calculés et mesurés, pour les 2 gammes de longueur d'onde, sont présentés en Figure $5 a$ et b. Dans les 2 cas, l'ajustement des calculs aux mesures est bon. Pour le rayonnement global, la droite de régression (Tableau IV) a cependant une pente significativement différente de 1 (mais une ordonnée à l'origine non différente de 0). Cette situation est principalement due à une sous-estimation du rayonnement transmis lorsque celui-ci est supérieur à $300 \mathrm{~W} \cdot \mathrm{m}^{-2}$. L'erreur quadratique moyenne est égale à $39 \mathrm{~W} \cdot \mathrm{m}^{-2}$, ce qui correspond en valeur relative à une erreur d'estimation de $12 \%$.

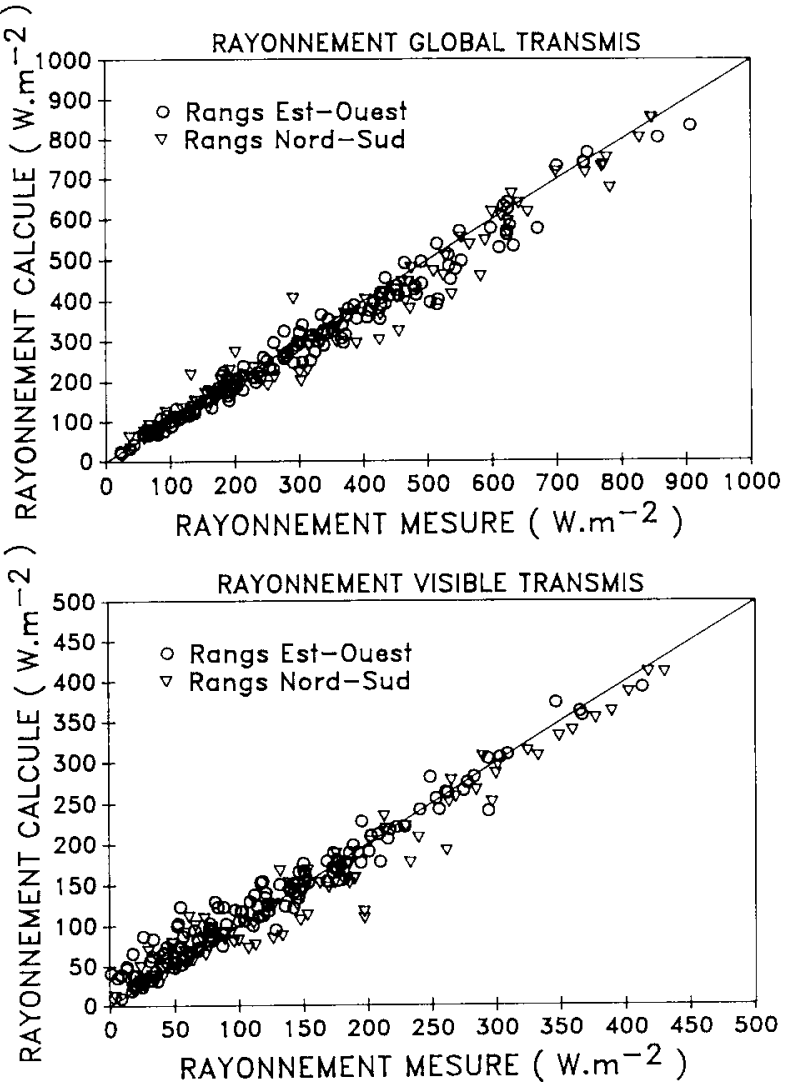

Fig. 5. Comparaison des rayonnements transmis calculés et mesurés. a. Rayonnement global. b. Rayonnement visible.

Dans le cas du rayonnement visible, la droite de régression (Tableau IV) est encore plus éloignée de la première bissectrice, bien que, de visu, l'ajustement des calculs aux mesures semble meilleur. Cela est dû à une surestimation des rayonnements inférieurs à $100 \mathrm{~W} \cdot \mathrm{m}^{-2}$, alors que les rayonnements plus élevés sont bien simulés. L'erreur quadratique moyenne reste

Tableau IV. Equation des droites de régression entre rayonnements calculés et mesurés.

\begin{tabular}{|c|c|c|c|c|}
\hline \multicolumn{5}{|l|}{ Ensemble des 2 cultures } \\
\hline$R_{\text {tot }}($ calc $)=0,873 R_{\text {tot }}$ & (mes) & 0,228 & $r^{2}=0,97$ & $N=270$ \\
\hline$R_{\text {vis }}($ calc $)=1,068 R_{\text {vis }}$ & (mes) & 1,180 & $r^{2}=0,84$ & $\mathrm{~N}=258$ \\
\hline$T_{\text {tot }}($ calc $)=0,928 T_{\text {tot }}$ & (mes) & 7,404 & $r^{2}=0,97$ & $N=252$ \\
\hline$T_{\text {vis }}($ calc $)=0,895 T_{\text {vis }}$ & (mes) & $+16,148$ & $r^{2}=0,95$ & $N=246$ \\
\hline \multicolumn{5}{|l|}{ Culture orienté est-ouest } \\
\hline$R_{\text {tot }}($ calc $)=0,851 R_{\text {tot }}$ & (mes) & 1,814 & $r^{2}=0,97$ & $N=150$ \\
\hline$R_{\text {vis }}($ calc $)=0,995 R_{\text {vis }}$ & (mes) & 1,038 & $r^{2}=0,85$ & $N=138$ \\
\hline $\mathrm{T}_{\text {tot }}($ calc $)=0,927 \mathrm{~T}_{\text {tot }}$ & (mes) & $+\quad 7,427$ & $r^{2}=0,97$ & $N=149$ \\
\hline$T_{\text {vis }}($ calc $)=0,909 T_{\text {vis }}$ & (mes) & $+20,290$ & $r^{2}=0,96$ & $N=136$ \\
\hline \multicolumn{5}{|l|}{ Culture orientée nord-sud } \\
\hline$R_{\text {tot }}($ calc $)=0,905 R_{\text {tot }}$ & (mes) & 2,039 & $r^{2}=0,97$ & $N=120$ \\
\hline$R_{\text {vis }}($ calc $)=1,210 R_{\text {vis }}$ & (mes) & 1,775 & $r^{2}=0,87$ & $\mathrm{~N}=120$ \\
\hline$T_{\text {tot }}($ calc $)=0,929 T_{\text {tot }}$ & (mes) & $+\quad 7,533$ & $r^{2}=0,97$ & $N=103$ \\
\hline$T_{\text {vis }}($ calc $)=0,896 T_{\text {vis }}$ & (mes) & $+8,956$ & $r^{2}=0,95$ & $\mathrm{~N}=110$ \\
\hline
\end{tabular}


cependant assez faible avec une valeur de 22 W. $\mathrm{m}^{-2}$.

\section{Comparaison des ajustements en fonction de l'orientation des rangs}

Les droites de régression, entre valeurs mesurées et calculées, ont été déterminées séparément pour les rangs orientés E-O et N-S (Tableau IV). Le test de Student, effectué sur les pentes et les coefficients constants de ces droites, a permis de comparer la qualité de l'ajustement du modèle aux mesures en fonction de l'orientation des rangs. Les coefficients $\mathrm{E}-\mathrm{O}$ et $\mathrm{N}$ $S$ ne sont pas significativement différents l'un de l'autre, sauf pour la pente de la droite dans le cas du rayonnement visible réfléchi. Compte tenu des remarques précédentes, cette unique discordance semble insuffisante pour dénoncer un mauvais fonctionnement du modèle dans la prise en compte de l'orientation de la culture.

Bien que satisfaisante, l'adéquation du modèle aux mesures devrait pouvoir être améliorée en réduisant certaines sources d'erreur : d'une part, l'estimation du rayonnement diffus incident est très approximative, et elle devrait être remplacée de façon avantageuse par une mesure au champ. D'autre part, la structure géométrique a été déterminée à partir d'un nombre limité de plantes (essentiellement à cause du coût temporel de cette mesure); l'incertitude sur cette mesure, qui peut être caractérisée par les intervalles de confiance des Figures 1 à 3 , est assez importante. Enfin, certains paramètres n'ont pas été mesurés, mais fixés à une valeur jugée réaliste; c'est le cas des propriétés optiques des feuilles et, dans une moindre mesure, du sol. Une analyse de sensibilité du modèle, prévue dans la poursuite de ce travail, devrait permettre de quantifier de façon plus précise l'influence de ces sources d'erreur.

\section{Discussion de la valeur du paramètre d'agen- cement des feuilles}

Le paramètre d'agencement du feuillage décrit, de manière globale, comment sont placés les éléments de surface foliaire les uns par rapport aux autres. II a une grande influence sur les sorties du modèle, car il intervient dans le calcul des fréquences de trous (Nilson, 1971). Connaissant la distribution des inclinaisons du feuillage, il peut être, dans le cas des couverts homogènes, déterminé à partir de photographies hémisphériques (Prévot, 1985).

Ce paramètre a été calé pour chaque date, et la Figure 6 montre l'évolution des valeurs optimisées au cours du développement de la culture. Lorsque le couvert est peu développé, une part

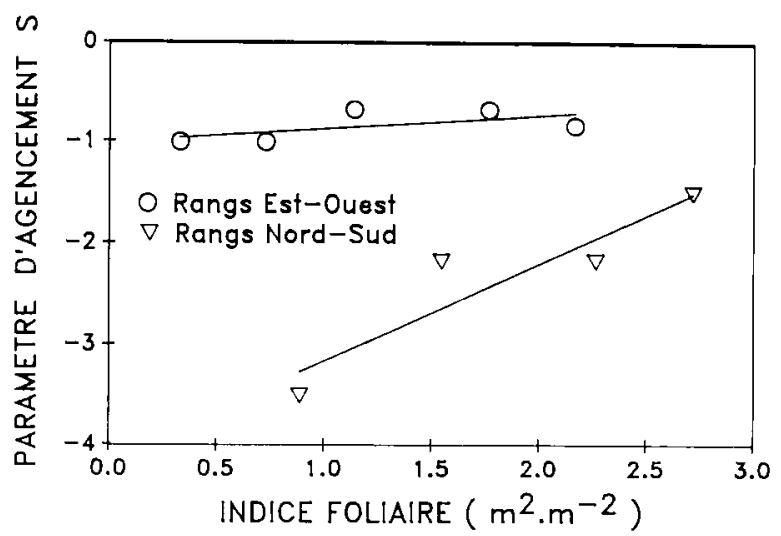

Fig. 6. Evolution du paramètre d'agencement du feuillage (valeur optimisée) en fonction de l'indice foliaire.

importante de l'ombrage des feuilles d'une plante est essentiellement due à la présence des feuilles supérieures de cette même plante. Compte tenu du port particulier du maïs, il semble cohérent d'observer des comportements fortement agrégatifs. En revanche, lorsque la culture se développe, une part de plus en plus importante de l'ombrage est due à la présence des feuilles des plantes des rangs voisins, dont la disposition par rapport aux feuilles d'une plante particulière est plus aléatoire.

Les différences observées au niveau de la structure géométrique permettent d'expliquer, au moins en partie, l'influence de l'orientation des rangs sur le paramètre d'agencement. Dans le cas de la culture orientée $E-O$, les feuilles ont tendance à s'étaler dans l'interrang sous l'action du vent, réduisant ainsi l'ombrage dû aux plantes voisines situées sur le même rang; ce n'est pas le cas des rangs $\mathrm{N}-\mathrm{S}$.

II paraît évident que cette analyse simple devrait être complétée par une étude de la variation spatiale de l'agencement des feuilles, qui devrait, a priori, être fortement agrégatif au centre du rang et plus aléatoire dans l'interrang.

II faut aussi noter qu'il existe une variation de ce paramètre selon l'axe vertical : Prévot (1985) a déterminé, pour un couvert de maïs bien développé, des valeurs variant de $-2,92$ pour la couche supérieure à $-0,25$ pour la couche intermédiaire et à $-1,04$ pour la couche inférieure. D'autre part, Nilson (1971) a indiqué que l'indice de dispersion pouvait varier en fonction de la direction des rayons. L'analyse des résidus (écarts entre valeurs calculées et mesurées) semble confirmer cette hypothèse :

- d'une part, les résidus sont plus étalés pour les faibles valeurs du rapport D/G (rapport du flux diffus incident au flux global incident) : Figure $7 \mathrm{a}$ et $b$ pour les rayonnements visibles réfléchis et transmis. Lorsque la contribution du flux direct au flux global est plus importante, la direction du 

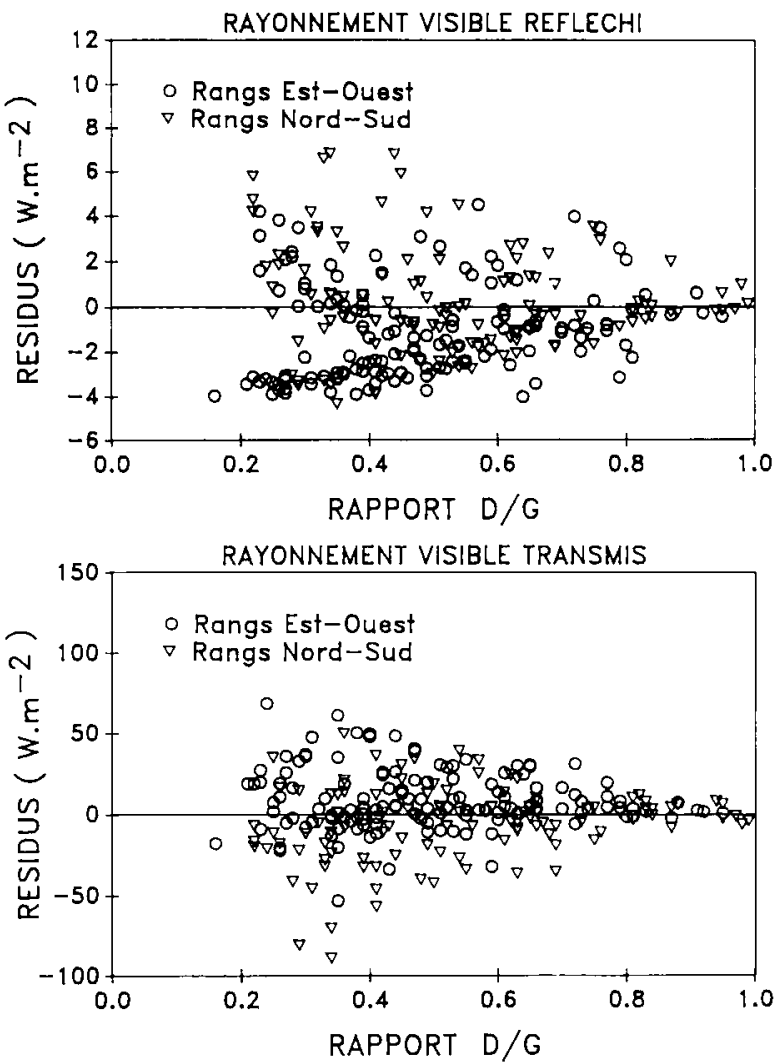

Fig. 7. Evolution des écarts entre valeurs calculées et mesurées en fonction du rapport $D / G$. a. Rayonnement visible réfléchi. b. Rayonnement visible transmis.

soleil est fortement privilégiée et les éventuelles variations du paramètre d'agencement en fonction de la direction du rayonnement, non prises en compte par le modèle, peuvent s'exprimer par des écarts plus importants entre calculs et mesures;

- d'autre part, les résidus sont liés à la hauteur du soleil. Dans le cas des rayonnements transmis global et visible, les coefficients de corrélation entre les résidus et la hauteur du soleil sont respectivement de $-0,374$ et $-0,469$. Ces valeurs négatives correspondent à une surestimation des rayonnements transmis pour les faibles hauteurs du soleil, et à une sous-estimation pour les hauteurs plus élevées. Elles semblent donc traduire un comportement agrégatif, qui augmente avec la hauteur des rayons incidents. Cela paraît correspondre au cas du maïs, dont le port favorise une superposition des feuilles plus particulièrement pour les directions verticales du rayonnement.

\section{Conclusion}

La validation, effectuée sur 2 cultures hétérogènes dans le plan horizontal, a permis de tester le modèle. Les valeurs calculées sont globalement en bon accord avec les mesures, et les écarts restent faibles. Le rayonnement global réfléchi est légèrement sous-estimé, alors que le rayonnement réfléchi visible est surestimé. Le rayonnement global transmis au sol est sousestimé pour les valeurs de rayonnement élevées, alors que le rayonnement visible transmis est surestimé pour les faibles valeurs de rayonnement. Les erreurs d'estimation varient de 10 à $20 \%$, en fonction du type de rayonnement, et l'ajustement des calculs aux mesures est pratiquement identique pour les rangs orientés $\mathrm{E}-\mathrm{O}$ et $\mathrm{N}-\mathrm{S}$. La structure géométrique du couvert est différente selon l'orientation des rangs, notamment à cause des conditions particulières de vent rencontrées à la Guadeloupe. Cela permet d'avancer une explication quant aux écarts rencontrés entre les 2 cultures au niveau des valeurs optimisées de l'indice de dispersion.

Il est à noter que la comparaison entre rayonnements calculés et mesurés a donné des résultats satisfaisants, que le couvert soit supposé homogène (Sinoquet, 1989) ou hétérogène. Dans les 2 cas, la structure géométrique de la culture n'était pas uniforme dans le plan horizontal. La suppression de l'hypothèse d'homogénéité ne semble donc pas nécessaire, dans le cas des cultures étudiées, si l'objectif est la détermination du bilan radiatif global de la culture. Cependant, les modèles appliqués aux couverts homogènes ne permettent pas d'étudier la distribution spatiale du rayonnement dans la culture ou au niveau du sol. Cela n'est possible qu'en utilisant un modèle qui tienne compte des variations spatiales de la structure géométrique du couvert dans le plan horizontal. C'est à ce type d'application que nous comptons utiliser ce modèle, notamment pour évaluer le rayonnement disponible dans l'interrang pour une culture associée de faible hauteur.

\section{Références}

Bonhomme R. (1983) Fonctionnement photosynthétique du couvert végétal In: Physiologie du Maïs (Gallais A., ed.). INRA, Paris, pp. 147-162

Bonhomme R. \& Varlet-Grancher C. (1977) Application aux couverts végétaux des lois de rayonnement en milieu diffusant. I. Etablissement des lois et vérifications expérimentales. Ann. Agron 28, 567-582

Bonhomme R. \& Varlet-Grancher C. (1978) Estimation of the gramineous crop geometry by plant profiles including leaf width variations. Photosynthetica (Prague) 12, 193-196

Lemeur R. \& Blad B.L. (1974) A critical review of ligth models for estimating the shortwave radiation regime of plants canopies. Agric. Meteorol. 14, 255-286

Loomis R.S. \& Williams W.A. (1969) Productivity and morphology of crops stands : patterns with leaves. In: Physiological Aspects of Crop Yield (Eastin J.D., Haskins F.A., Sullivan C.Y. \& Van Bavel C.H.M., eds). Am. Soc. Agron., Madison, Wisc., pp. 27-47 
Mure V. (1984) Influence de l'orientation des rangs sur le microclimat et le développement de cultures discontinues de maïs en zone tropicale. Mémoire de fin d'études CNEARC

Myneni R.B., Asrar G., Kanemasu E.T., Lawlor D.J. \& Impens I. (1986) Canopy architecture, irradiance distribution on leaf surfaces and consequent photosynthetic efficiencies in heterogeneous plant canopies. Part I. Theoretical considerations. Agric. For. Meteorol. 37, 189-204

Nilson T. (1971) A theoretical analysis of the frequency of gaps in plants stands, Agric. Meteorol. 8, 25-38

Ozisik N.M. (1981) Radiative Transfer. Wiley Intersc., New York

Prévot L. (1985) Modélisation des échanges radiatifs au sein des couverts végétaux, application à la télédétection, validation sur un couvert de maïs. Thèse, Paris VI

Ross J. (1981) The Radiation Regime and Architecture of Plants Stands. Dr. W. Junk pub., The Netherlands
Ross J. \& Nilson T. (1967a) Spatial orientation of leaves in plant canopy and its determination. In: Photosynthesis of Productive Systems (Nichiporovitch A.A., ed.). Engl. Transl. Israël Prog. Sci. Transl., Jerusalem, pp. 86-99

Ross J. \& Nilson T. (1967b) The vertical distribution of biomass in crop stand. In: Photosynthesis of Productive Systems (Nichiporovitch A.A., ed.).Engl. Transl. Israël Prog. Sci. Transl., Jerusalem, pp. 75-85

Sinoquet H. (1985) Programme régional maîtrise de l'énergie : évaluation des composantes du rayonnement solaire en Guadeloupe. Internal note, INRA Guadeloupe

Sinoquet H. (1989) Modélisation de l'interception des rayonnements solaires dans une culture en rang. 1. Aspects théoriques. Agronomie 9, 125-135

Varlet-Grancher C. (1975) Variation et estimation de l'énergie d'origine solaire reçue sur des plans d'inclinaison et d'azimut variables. Ann. Agron. 26, 245-264 